\title{
A prospective association between dietary folate intake and type 2 diabetes risk among Korean adults aged 40 years or older: the Korean Multi-Rural Communities Cohort (MRCohort) Study
}

\author{
Sang M. Hong ${ }^{1}$, Hey W. Woo, ${ }^{2,3}$, Mi K. Kim ${ }^{2,3 *}$, Se Y. Kim²,3, Young-Hoon Lee ${ }^{4}$, Dong H. Shin ${ }^{5}$, \\ Min-Ho Shin ${ }^{6}$, Byung-Yeol Chun ${ }^{7}$ and Bo Y. Choi ${ }^{2,3}$ \\ ${ }^{1}$ Department of Internal Medicine, Division of Endocrinology, Keunjaebong-gil, Hallym University Dongtan Sacred Heart \\ Hospital 7, Hwaseong-si Gyeonggi-do, 18450, South Korea \\ ${ }^{2}$ Department of Preventive Medicine, College of Medicine, Hanyang University, Seoul, O4763, South Korea \\ ${ }^{3}$ Institute for Health and Society, Hanyang University, Seoul, 04763, South Korea \\ ${ }^{4}$ Department of Preventive Medicine E Institute of Wonkwang Medical Science, Wonkwang University College of Medicine, \\ Iksan, 54538, South Korea \\ ${ }^{5}$ Department of Occupational and Environmental Medicine, Keimyung University Dongsan Medical Center, Daegu, 42403, \\ 42403, South Korea \\ ${ }^{6}$ Department of Preventive Medicine, Chonnam National University Medical School, Gwangju, 61186, South Korea \\ ${ }^{7}$ Department of Preventive Medicine, School of Medicine, and Health Promotion Research Center, Kyungpook National \\ University, Daegu, 41566, South Korea
}

(Submitted 21 September 2016 - Final revision received 8 September 2017 - Accepted 25 September 2017 - First published online 4 December 2017)

\section{Abstract}

It has not been well established whether dietary folate intake reduces the risk of diabetes development. We aimed to clarify the prospective association between dietary folate intake and type 2 diabetes (T2D) risk among 7333 Korean adults aged 40 years or older who were included in the Multi-Rural Communities Cohort. Dietary folate intake was estimated from all 106 food items listed on a FFQ, not including folate intake from supplements. Two different measurements of dietary folate intake were used: the baseline consumption and the average consumption from baseline until just before the end of follow-up. The association between folate intake and T2D risk was determined through a modified Poisson regression model with a robust error estimator controlling for potential confounders. For 29745 person years, 319 cases of diabetes were ascertained. In multivariable analyses, dietary folate intake was inversely associated with risk of T2D for women, not for men. For women, the incidence rate ratio of diabetes in the third tertile compared with the first tertile was $0.57\left(95 \% \mathrm{CI} 0 \cdot 38-0 \cdot 87, P_{\text {for trend }}=0 \cdot 0085\right)$ in the baseline consumption model and $0.64\left(95 \%\right.$ CI $\left.0.43-0.95, P_{\text {for trend }}=0.0244\right)$ in the average consumption model. These inverse associations was found in both normal fasting blood glucose group and impaired fasting glucose group among women. Among non-users of multinutrients and vitamin supplements, the significant inverse association remained. Thus, higher dietary intake of folate is prospectively associated with lower risk of diabetes for women.

Key words: Dietary folate intakes: Type 2 diabetes: Prospective cohort studies: Korea

Folate is a water-soluble $\mathrm{B}$ vitamin that is naturally present in some foods (polyglutamate form), especially fruits, vegetables (particularly dark green leafy vegetables), legumes, dairy products, poultry and meat and that is also available as a dietary supplement (monoglutamate form, folic acid) ${ }^{(1)}$. Folate has a crucial role on one- $\mathrm{C}$ metabolism as one-C acceptor and donor and is also well known to act as a coenzyme, which may be involved in pyrimidine and purine synthesis and various methylation reactions ${ }^{(2,3)}$.
Low folate status has been associated with an increased risk of chronic diseases such as $\mathrm{CVD}^{(4)}$, cancer $^{(5)}$ and cognitive impairment ${ }^{(6)}$. Hyperhomocysteinemia, which is positively related to folate deficiency, has been also associated with increased risk of CVD and cerebrovascular diseases in epidemiological studies ${ }^{(7,8)}$, although a meta-analysis of $\mathrm{B}$ vitamin supplementation including folate showed no reduction of the risk of vascular diseases ${ }^{(9)}$. Diabetes is a well-known risk factor for the development of CVD and cerebrovascular diseases ${ }^{(10)}$

Abbreviations: FBG, fasting blood glucose; IFG, impaired fasting glucose; IRR, incidence rate ratio; NFG, normal fasting glucose; T2D, type 2 diabetes. 
and is affected by environmental factors as well as genetic factors $^{(11)}$. Previous meta-analyses showed the epidemiological evidence for associations of type 2 diabetes (T2D) with education level $^{(12)}$, smoking status ${ }^{(13)}$, drinking status ${ }^{(14)}$, regular exercise ${ }^{(15)}$, BMI/waist circumference (WC) ${ }^{(16)}$, and some dietary components such as processed red meat, sugarsweetened beverage, cereal fibre and whole grains, etc. ${ }^{(17)}$. Among dietary factors, epidemiological evidence regarding the relationship between dietary folate intake and T2D is lacking. However, in a meta-analysis ${ }^{(18)}$, the negative association between a methylene-tetrahydrofolate reductase (MTHFR) variant $(677 \mathrm{C}>\mathrm{T})$ and the development of $\mathrm{T} 2 \mathrm{D}$ suggested the beneficial effect of folate on T2D.

Until recently, there has not been a prospective study evaluating whether higher dietary folate intake is associated with a lesser chance of developing diabetes. Therefore, we examined the prospective association between folate intake and T2D risk among men and women aged 40 years or older in a community-based cohort in South Korea.

\section{Methods}

\section{Study design and population}

This prospective cohort study was the ongoing Multi-Rural Communities Cohort, part of the Korean Genome and Epidemiology Study (KoGES). Participants aged 40 years or over ( $n$ 9692) were recruited from three rural areas in the Republic of Korea - Yangpyeong, Namwon and Goryeong between January 2005 and December 2009 for an investigation of CVD risk factors. Follow-up surveys for re-examination similar to the baseline examination were conducted every 2-4 years (average 2.85 years) after the baseline survey. Participants returned for a second examination between 2007 and 2013 (mean of follow-up duration, $2 \cdot 5$ (sD 1.0) years) and for a third examination between 2010 and 2013 (mean of follow-up duration, 3.2 (sD 1.0) years). Of total participants ( $n$ 9692), 7640 participants $(78.0 \%)$ for the second examination and 4040 participants $(41.7 \%)$ for the third examination completed. Average follow-up years were 4.06 (sD 1.96) years. Participants who had a history of heart disease, stroke and/or cancer ( $n$ 1116); who had been treated with anti-diabetic drugs or insulin; or whose fasting blood glucose (FBG) level at baseline was $7.0 \mathrm{mmol} / \mathrm{l}(126 \mathrm{mg} / \mathrm{dl})$ or higher $(n$ 976) were excluded. In addition, those with implausible dietary intakes $(<2092$ or $>16736 \mathrm{~kJ} / \mathrm{d}$ ( $<500$ or $>4000 \mathrm{kcal} / \mathrm{d}$ ) or $>10$ missing food items; $n 77$ ), with insufficient blood specimens, or with missing data for important covariates needed to identify the association between diabetes and folate intake ( $n$ 190) were removed. Thus, 7333 participants (2693 men and 4640 women) were included in the final analysis. This study was conducted according to the guidelines of the Declaration of Helsinki and was approved by the Institutional Review Boards of Hanyang University, Chonnam National University and Keimyeong University.

\section{Dietary assessment}

To assess dietary nutrient intake, we used a previously validated semi-quantitative FFQ composed of 106 food items ${ }^{(19)}$.
Trained interviewers asked participants to report how often they had consumed each item over the past year, using nine frequency categories ranging from 'never or rarely' to ' 3 times/d,' as well as three standardised portion sizes. In the case of limited seasonal food items, additional data were collected on how long these items were eaten (for 3, 6, 9 or 12 months of the year). Dietary nutrient intakes, including folate intake, were calculated by multiplying the reported intake frequency of each food by the amount of nutrients in a serving size of that food based on nutrient database of CAN-PRO 4.0 (Computer Aided Nutritional analysis program) of the Korean Nutrition Society ${ }^{(20)}$. For seasonal foods, seasonal intake period was additionally considered to calculate nutrient intake. To isolate variation in nutrient intake due only to the nutrient composition of the diet from extraneous variation by the correlation with total energy intake $^{(21)}$, total energy-adjusted-nutrient intakes were estimated by residual method, which uses the regression model with total energy intake as the independent variable and nutrient intake as the dependent variable. Nutrients intake from supplements could not be calculated, because there was no publicly available wellestablished national database and because most participants did not correctly recall the brand name.

\section{Assessment of non-dietary factors}

Data were collected from questionnaires and examinations according to standard protocols and procedures. Study participants were asked about demographic information including age, education level, smoking status, alcohol consumption, physical activity, medical history, menopause status and supplement use by trained interviewers. For anthropometric assessment, height and weight were measured to the nearest $0 \cdot 1 \mathrm{~cm}$ and $0 \cdot 1 \mathrm{~kg}$, respectively, whereas subjects were dressed in light clothing. BMI was calculated as the ratio of weight $(\mathrm{kg})$ to squared height $\left(\mathrm{m}^{2}\right)$. WC was measured at the midpoint between the lowest rib margin and the iliac crest. Systolic and diastolic blood pressure were measured after $>5 \mathrm{~min}$ of rest in the seated position, by means of a standard sphygmomanometer and a cuff on the right arm. Blood pressure was measured at least twice, at 5-min intervals, and the last two measurements were averaged for the analysis. From blood samples collected after an 8-h fast, FBG levels was analysed on an ADVIA1650 Automatic Analyzer (Siemens).

\section{Ascertainment of type 2 diabetes}

Participants were asked to report a new diagnosis of diabetes at every visit. Those reporting physician-diagnosed diabetes were asked whether they were being treated with oral anti-diabetic medication and/or insulin. Based on the criteria of the American Diabetes Association $^{(22)}$, subjects were confirmed to have diabetes if they exhibited at least one of the following: (1) elevated FBG $\geq 126 \mathrm{mg} / \mathrm{dl}(7.0 \mathrm{mmol} / \mathrm{l})$ or (2) treatment with hypoglycaemic medication (insulin or oral drugs).

\section{Statistical analysis}

We calculated the person time for each participant from the baseline examination date until T2D diagnosis, death from any 
cause or censoring. If participants were lost to follow-up, their follow-up times were assigned as half the median of the person years of participants who properly completed follow-up ${ }^{(23)}$. We analysed the association of diabetes with two different measures of folate intake, baseline folate consumption and average folate consumption. The latter might reduce measurement error and/or more effectively represent long-term dietary patterns, because measured changes in diets of individuals over time are a mix of true variation and measurement error ${ }^{(17)}$. The average dietary consumption of folate was calculated by averaging the intake at baseline and at repeated examination just before the censoring time or endpoint of each subject. The general characteristics of the participants were described as means and standard deviations for continuous variables and as numbers and percentages for categorical variables.

Participants were divided into tertiles of folate intake. The low intake group, moderate intake group and high intake group were presented as the first tertile (T1), the second tertile (T2) and the third tertile (T3), respectively. Potential confounding factors were selected based on previously reported association with T2D in the meta-analysis using prospective cohort results (education level ${ }^{(12)}$, smoking status ${ }^{(13)}$, drinking status ${ }^{(14)}$, regular exercise $\mathrm{e}^{(15)}$ and $\left.\mathrm{BMI} / \mathrm{WC}^{(16)}\right)$. Likewise, for dietary factors, nutrients and foods previously reporting the significant association with T2D in the meta-analysis of prospective cohort studies (haeme-Fe, glycaemic index/glycaemic load, Mg, cereal fibre, processed meat, unprocessed red meat, white rice, vegetables, dairy product, sweetened beverages and coffee consumption) ${ }^{(17)}$ and randomised clinical trial (total energy intake ${ }^{(24)}$ ) were first considered as potential confounders. However, haeme-Fe and cereal fibre could not be calculated because of lacking of database. Fe from animal foods and fibre from cereals and grains were used. Among those factors, factors showing statistically significant difference (Tukey's multiple comparison) or trend according to the tertiles of folate intake were selected using the general linear model after controlling for age. For the test of linear trends, the median folate intake values of the tertiles were treated as continuous variables, and the level of significance was set at $0 \cdot 05$. To reduce collinearity among covariates, the following steps were taken; First, age-adjusted bivariate correlations were evaluated among covariates. Then, if covariates were highly correlated with dietary folate intake at Pearson's $r \geq 0 \cdot 6$, they were not included in the final analysis and if there was high correlation between two covariates, we decided whether to select which covariate as a potential confounder. Next, among nutrients and foods that may reflect the same aspects of diet such as carbohydrate, glycaemic index, glycaemic load and white rice, only one variable was selected. Because subjects may have modified their diets after recognising their own relatively high FBG level and the follow-up period in the present study was relatively short, we adjusted for baseline FBG. In addition, the modified Alternative Healthy Eating Index ${ }^{(25)}$ using Korean Dietary Reference Intakes (KAHEI) was also included to reduce confounding effect due to the relation to a healthy diet of dietary folate intake.

To estimate the incidence rate ratios (IRR) and 95\% CI according to tertile of dietary folate intake, we used a modified
Poisson regression model with a robust error estimator ${ }^{(26,27)}$. The age-adjusted model and the multivariable-adjusted models were employed. We could not isolate prediabetes due to no oral glucose tolerance test (OGTT) data in the present study, but it could be confirmed that there were just a few prediabetes by OGTT among participants with FBG $<100 \mathrm{mg} / \mathrm{dl}$ $(<5.6 \mathrm{mmol} / \mathrm{l}, 3 \cdot 13 \%$ in another community cohort, a part of KoGES). Therefore, the association between dietary folate intake and T2D were separately analysed in normal fasting glucose (NFG) group with $<100 \mathrm{mg} / \mathrm{dl} \mathrm{FBG}$ value $(5.6 \mathrm{mmol} / \mathrm{l})$ and in impaired fasting glucose (IFG) group which was defined as $\geq 100$ and $<126 \mathrm{mg} / \mathrm{dl}$ of FBG value $(5.6-6.9 \mathrm{mmol} / \mathrm{l})$ by the American Diabetes Association ${ }^{(22)}$. The interaction of dietary folate intake with FBG groups and sex were evaluated in the modified Poisson regression model. Taking nutrient supplements is a health-related behaviour and also provides folic acid ${ }^{(28)}$. Therefore, we additionally analysed the results among supplement non-users after excluding users of only multinutrients and after additionally excluding users of vitamin supplements such as vitamin $\mathrm{A}$, vitamin $\mathrm{C}, \beta$-carotene, vitamin $\mathrm{B}$ complex and vitamin $\mathrm{E}$.

For sensitivity analysis, the analyses using two different person times assignment to loss to follow-up were conducted ${ }^{(23)}$. The first assignment was based on the assumption that all persons were lost immediately after the last date they were known to be present, and the second was based on the assumption that they were all lost immediately before the first date on which they were known to be absent. At the second assignment, we assigned the median person time between visits. It assumed that the correct rate lied somewhere between two rates. Furthermore, a possible difference in the whole dietary patterns between the lowest and highest tertile of dietary folate was investigated using radar charts ${ }^{(29,30)}$. To illustrate radar chart, first, twenty-two food groups (fruit, mushroom, seaweed, poultry, unprocessed red meat, processed red meat, fish/seafood/shell, egg, dairy products, pulses/soya, nut/seed, coffee, snack/sugar, sugar-sweetened beverage, alcohol consumption, whole grain, refined grain, noodle/bred, potato/ starch, green leafy vegetable, other vegetables and salted vegetable) were calculated using foods in recipe of the FFQ. The median intake $(\mathrm{g} / \mathrm{d})$ of each food group for participants in T1 (or T3) of folate intake was compared with their median intake values for the entire population to obtain the relative percentage of food or beverage intake. Radar chart was illustrated using those relative percentages for the baseline and the average folate intake among men and women. The intake of food groups $(\mathrm{g} / \mathrm{d})$ was energy-adjusted value using the residual method $^{(21)}$. SAS software (version 9.4; SAS Institute Inc.) and R-software (version 3.4.0, http://www.r-project.org) were used for all statistical analyses.

\section{Results}

The study participants included 2693 men (36.7\%) and 4640 women $(63.3 \%)$. The average age was 62.0 (SD 9.7) years among men and 60.7 (SD 9.9) years among women. Men were more likely to be current drinkers $(66 \cdot 7 v .30 \cdot 2 \%)$ and current smokers $(35.8 v .3 .6 \%)$ and have higher education (29.6 $v$. $14.3 \%)$ than women. Table 1 displays the age-adjusted 
Table 1. Age-adjusted characteristics of the study participants according to tertiles (T) of folate intake at baseline survey (Mean values with their standard errors; numbers and percentages)

\begin{tabular}{|c|c|c|c|c|c|c|c|c|}
\hline \multirow[b]{3}{*}{ Characteristics } & \multicolumn{6}{|c|}{ Dietary folate intake $(\mu \mathrm{g} / \mathrm{d})$} & \multirow[b]{3}{*}{$P_{\text {difference }}$} & \multirow[b]{3}{*}{$P_{\text {trend }}$} \\
\hline & \multicolumn{2}{|c|}{$\mathrm{T} 1$} & \multicolumn{2}{|c|}{ T2 } & \multicolumn{2}{|c|}{ T3 } & & \\
\hline & Mean & SE & Mean & SE & Mean & SE & & \\
\hline \multicolumn{9}{|l|}{ Men ( $n$ 2693) } \\
\hline$n$ & \multicolumn{2}{|c|}{897} & \multicolumn{2}{|c|}{898} & \multicolumn{2}{|c|}{898} & & \\
\hline Median intake & & & & & & & & \\
\hline Min, $\max$ & $77 \cdot 8$ & & 317 & $26 \cdot 2$ & $426 \cdot 8$ & $2 \cdot 9$ & & \\
\hline Age (years) & 62.5 & 0.33 & $61 \cdot 8$ & 0.32 & 61.6 & 0.32 & 0.1324 & 0.0691 \\
\hline Higher education $(\%)^{\star}$ & & & & & & & 0.0062 & 0.0016 \\
\hline$n$ & & & & & & & & \\
\hline$\%$ & & & & & & & & \\
\hline Smoking status (\%) & & & & & & & & \\
\hline Former smoker & & & & & & & 0.0022 & 0.0125 \\
\hline$n$ & & & & & & & & \\
\hline$\%$ & & & & & & & & \\
\hline Current smoker & & & & & & & 0.0004 & 0.0272 \\
\hline$n$ & & & & & & & & \\
\hline$\%$ & & & & & & & & \\
\hline Drinking status (\%) & & & & & & & & \\
\hline Former drinker & & & & & & & 0.5125 & 0.8075 \\
\hline$n$ & & & & & & & & \\
\hline$\%$ & & & & & & & & \\
\hline Current drinker & & & & & & & 0.9472 & 0.8047 \\
\hline$n$ & & & & & & & & \\
\hline$\%$ & & & & & & & & \\
\hline Alcohol consumption (ml/d) & 31.9 & 1.86 & 30.5 & 1.86 & $28 \cdot 9$ & 1.86 & 0.5173 & 0.2511 \\
\hline Regular exercise $(\%) \dagger$ & . & . & & & & & 0.0005 & 0.0028 \\
\hline$n$ & & & & & & & & \\
\hline$\%$ & & & & & & & & \\
\hline $\operatorname{BMI}\left(\mathrm{kg} / \mathrm{m}^{2}\right)$ & $23 \cdot 7$ & 0.09 & 23.9 & 0.09 & 23.9 & 0.09 & 0.1210 & 0.0755 \\
\hline $\mathrm{BMI} \geq 25 \mathrm{~kg} / \mathrm{m}^{2}(\%)$ & 290 & $32 \cdot 9$ & 324 & $35 \cdot 9$ & 342 & $35 \cdot 7$ & 0.3091 & 0.2180 \\
\hline Waist circumference $(\mathrm{cm})$ & $84 \cdot 3^{\mathrm{a}}$ & 0.27 & $85 \cdot 4^{\mathrm{b}}$ & 0.27 & $85 \cdot 3^{b, c}$ & 0.27 & 0.0095 & 0.0183 \\
\hline Fasting blood glucose (mg/dl) & $96 \cdot 2^{\mathrm{a}}$ & 0.34 & $97 \cdot 1^{\mathrm{a}}$ & 0.33 & $97 \cdot 6^{\mathrm{b}}$ & 0.33 & 0.0164 & 0.0057 \\
\hline Impaired fasting glucose (\%) & & & & & & & 0.0225 & 0.0060 \\
\hline$n$ & & & & & & & & \\
\hline$\%$ & & & & & & & & \\
\hline Dietary intakeł & & & & & & & & \\
\hline Energy $(\mathrm{kJ} / \mathrm{d})$ & $6906 \cdot 9^{a}$ & $64 \cdot 8$ & $6968 \cdot 9^{\mathrm{a}}$ & $64 \cdot 8$ & $6750 \cdot 0^{\mathrm{b}}$ & $64 \cdot 8$ & 0.0485 & 0.0674 \\
\hline Carbohydrate $(\mathrm{g} / \mathrm{d})$ & $325 \cdot 3$ & 1.05 & 323.6 & 1.05 & $321 \cdot 7$ & 1.05 & 0.0548 & 0.0160 \\
\hline Protein $(\mathrm{g} / \mathrm{d})$ & $47 \cdot 8^{\mathrm{a}}$ & 0.27 & $52 \cdot 3^{\mathrm{b}}$ & 0.27 & $57.02^{\mathrm{C}}$ & 0.27 & $<0.0001$ & $<0.0001$ \\
\hline Fat $(g / d)$ & $20 \cdot 3^{a}$ & 0.29 & $21 \cdot 4^{\mathrm{b}}$ & 0.29 & $22 \cdot 1^{\mathrm{b}}$ & 0.29 & $<0.0001$ & $<0.0001$ \\
\hline Fe from animal food $(\mathrm{mg} / \mathrm{d})$ & $1 \cdot 3^{\mathrm{a}}$ & 0.04 & $1.6^{\mathrm{b}}$ & 0.04 & $1.9^{\mathrm{C}}$ & 0.04 & $<0.0001$ & $<0.0001$ \\
\hline Glycaemic index & $59.0^{a}$ & 0.08 & $57 \cdot 6^{\mathrm{b}}$ & 0.08 & $55 \cdot 6^{\mathrm{C}}$ & 0.08 & $<0.0001$ & $<0.0001$ \\
\hline Glycaemic load & $192 \cdot 0^{\mathrm{a}}$ & 0.77 & $186 \cdot 7^{\mathrm{b}}$ & 0.77 & $179 \cdot 2^{c}$ & 0.77 & $<0.0001$ & $<0.0001$ \\
\hline $\mathrm{Mg}(\mathrm{mg} / \mathrm{d})$ & $89 \cdot 1^{\mathrm{a}}$ & 0.63 & $91^{\mathrm{a}}$ & 0.63 & $96 \cdot 9^{c}$ & 0.63 & $<0.0001$ & $<0.0001$ \\
\hline Fibre from cereals and grains $(\mathrm{g} / \mathrm{d})$ & $4 \cdot 8$ & 0.05 & $4 \cdot 8$ & 0.05 & 4.7 & 0.05 & 0.3945 & 0.5461 \\
\hline Supplement users & & & & & & & & \\
\hline Multinutrients (\%) & & & & & & & 0.0070 & 0.0022 \\
\hline$n$ & & & & & & & & \\
\hline$\%$ & & & & & & & & \\
\hline Multinutrients or vitamin supplements (\%)§ & & & & & & & 0.0010 & 0.0002 \\
\hline$n$ & & & & & & & & \\
\hline$\%$ & & & & & & & & \\
\hline Food groupll & & & & & & & & \\
\hline Total vegetable $(\mathrm{g} / \mathrm{d})$ & $42 \cdot 7^{\mathrm{a}}$ & 1.63 & $58.0^{\mathrm{b}}$ & 1.63 & $89 \cdot 7^{\mathrm{C}}$ & 1.63 & $<0.0001$ & $<0.0001$ \\
\hline White rice $(\mathrm{g} / \mathrm{d})$ & $702 \cdot 9^{\mathrm{a}}$ & 4.89 & $663 \cdot 0^{\mathrm{a}}$ & 4.89 & $628 \cdot 2^{\mathrm{b}}$ & 4.89 & 0.0018 & $<0.0001$ \\
\hline Dairy products $(g / d)$ & $62 \cdot 3^{\mathrm{a}}$ & 3.49 & $85 \cdot 0^{\mathrm{b}}$ & 3.49 & $83 \cdot 5^{\mathrm{b}}$ & 3.49 & $<0.0001$ & $<0.0001$ \\
\hline Red meat $(\mathrm{g} / \mathrm{d})$ & $33 \cdot 4^{\mathrm{a}}$ & $1 \cdot 1$ & $30 \cdot 8^{a}$ & $1 \cdot 1$ & $27 \cdot 8^{\mathrm{b}}$ & $1 \cdot 1$ & 0.0017 & 0.0004 \\
\hline Processed meat $(\mathrm{g} / \mathrm{d})$ & 0.3 & 0.06 & 0.4 & 0.06 & 0.4 & 0.06 & 0.3224 & 0.3332 \\
\hline Sweetened beverages $(\mathrm{g} / \mathrm{d})$ & $20 \cdot 3^{\mathrm{a}}$ & 1.98 & $17 \cdot 4$ & 1.98 & 14.5 & 1.98 & 0.1253 & 0.0420 \\
\hline Coffee $(g / d)$ & $4 \cdot 5^{a}$ & 0.13 & $4 \cdot 1^{\mathrm{a}}$ & 0.13 & $3.9^{b}$ & 0.13 & 0.0158 & 0.0049 \\
\hline Dietary quality score & & & & & & & & \\
\hline KAHEIף & $31.4^{a}$ & 0.24 & $33.9^{\mathrm{b}}$ & 0.24 & $36 \cdot 3^{b}$ & 0.24 & $<0.0001$ & $<0.0001$ \\
\hline Women ( $n$ 4640) & & & & & & & & \\
\hline$n$ & 15 & & & & & & & \\
\hline Median intake & & & & & & & & \\
\hline Min, $\max$ & 97.5 & & 285 & $30 \cdot 6$ & $380 \cdot 7$ & & & \\
\hline Age (years) & $62 \cdot 8^{\mathrm{a}}$ & 0.25 & $60 \cdot 6^{\mathrm{b}}$ & 0.25 & $58 \cdot 7^{\mathrm{C}}$ & 0.25 & $<0.0001$ & $<0.0001$ \\
\hline
\end{tabular}


Table 1. Continued

\begin{tabular}{|c|c|c|c|c|c|c|c|c|}
\hline \multirow[b]{3}{*}{ Characteristics } & \multicolumn{6}{|c|}{ Dietary folate intake $(\mu \mathrm{g} / \mathrm{d})$} & \multirow[b]{3}{*}{$P_{\text {difference }}$} & \multirow[b]{3}{*}{$P_{\text {trend }}$} \\
\hline & \multicolumn{2}{|c|}{$\mathrm{T} 1$} & \multicolumn{2}{|c|}{$\mathrm{T} 2$} & \multicolumn{2}{|c|}{ T3 } & & \\
\hline & Mean & SE & Mean & SE & Mean & SE & & \\
\hline Higher education (\%)* & \multirow{2}{*}{\multicolumn{2}{|c|}{137}} & \multirow{2}{*}{\multicolumn{2}{|c|}{223}} & & & 0.0009 & 0.0002 \\
\hline n & & & & & \multicolumn{2}{|c|}{301} & & \\
\hline$\%$ & \multicolumn{2}{|c|}{$12 \cdot 1^{\mathrm{a}}$} & \multicolumn{2}{|c|}{$14 \cdot 3^{a}$} & \multicolumn{2}{|c|}{$16 \cdot 4^{\mathrm{b}}$} & & \\
\hline \multicolumn{9}{|l|}{ Smoking status (\%) } \\
\hline Former smoker & & & & & & & 0.2823 & 0.2595 \\
\hline$n$ & & & & & & & & \\
\hline$\%$ & & & & & & & & \\
\hline Current smoker & & & & & & & 0.0234 & 0.1380 \\
\hline$n$ & & & & & & & & \\
\hline$\%$ & & & & & & & & \\
\hline Drinking status (\%) & & & & & & & & \\
\hline Former drinker & & & & & & & 0.4162 & 0.1935 \\
\hline$n$ & & & & & & & & \\
\hline$\%$ & & & & & & & & \\
\hline Current drinker & & & & & & & 0.3099 & 0.1602 \\
\hline$n$ & & & & & & & & \\
\hline$\%$ & & & & & & & & \\
\hline Alcohol consumption $(\mathrm{ml} / \mathrm{d})$ & $2 \cdot 0^{a}$ & 0.32 & $2 \cdot 1^{\mathrm{a}}$ & 0.31 & $3.3^{\mathrm{b}}$ & 0.32 & 0.0080 & 0.0046 \\
\hline Regular exercise $(\%) \dagger$ & & & & & & & 0.0006 & 0.0003 \\
\hline$n$ & & & & & & & & \\
\hline$\%$ & & & & & & & & \\
\hline BMI $\left(\mathrm{kg} / \mathrm{m}^{2}\right)$ & $24 \cdot 3$ & 0.08 & 24.4 & 0.08 & 24.5 & 0.08 & $0 \cdot 1663$ & 0.0593 \\
\hline $\mathrm{BMI} \geq 25 \mathrm{~kg} / \mathrm{m}^{2}(\%)$ & 583 & 38.4 & 632 & 40.8 & 654 & $41 \cdot 7$ & 0.1564 & 0.0702 \\
\hline Waist circumference $(\mathrm{cm})$ & $82 \cdot 4^{\mathrm{a}}$ & 0.23 & $83.4^{\mathrm{b}}$ & 0.23 & $83.5^{\mathrm{b}}$ & 0.23 & 0.0007 & 0.0009 \\
\hline Fasting blood glucose (mg/dl) & $93 \cdot 5^{\mathrm{a}}$ & 0.24 & $94 \cdot 2^{\mathrm{a}, \mathrm{b}}$ & 0.24 & $94 \cdot 8^{\mathrm{b}, \mathrm{c}}$ & 0.24 & 0.0010 & 0.0002 \\
\hline Impaired fasting glucose (\%) & & & & & & & 0.0368 & 0.0174 \\
\hline$n$ & & & & & & & & \\
\hline$\%$ & & & & & & & & \\
\hline Menopause, among women (\%) & & & & & & & 0.9335 & 0.9626 \\
\hline$n$ & & & & & & & & \\
\hline$\%$ & & & & & & & & \\
\hline Dietary intakeł & & & & & & & & \\
\hline Energy $(\mathrm{kJ} / \mathrm{d})$ & $6143 \cdot 1^{a}$ & $43 \cdot 2$ & $6080 \cdot 3^{a}$ & $42 \cdot 9$ & $5742 \cdot 1^{\mathrm{b}}$ & $43 \cdot 2$ & $<0.0001$ & $<0.0001$ \\
\hline Carbohydrate $(\mathrm{g} / \mathrm{d})$ & $298 \cdot 9^{\mathrm{a}}$ & 0.66 & $294.2^{b}$ & 0.65 & $288.5^{\mathrm{c}}$ & 0.66 & $<0.0001$ & $<0.0001$ \\
\hline Protein $(\mathrm{g} / \mathrm{d})$ & $39.2^{a}$ & 0.17 & $43.2^{\mathrm{b}}$ & 0.16 & $48 \cdot 1^{\mathrm{c}}$ & 0.17 & $<0.0001$ & $<0.0001$ \\
\hline Fat $(\mathrm{g} / \mathrm{d})$ & $12 \cdot 6^{a}$ & 0.17 & $14 \cdot 3^{b}$ & 0.17 & $16 \cdot 2^{\mathrm{c}}$ & $0 \cdot 17$ & $<0.0001$ & $<0.0001$ \\
\hline Fe from animal food $(\mathrm{mg} / \mathrm{d})$ & $0.9^{\mathrm{a}}$ & 0.02 & $1.2^{\mathrm{b}}$ & 0.02 & $1.6^{\mathrm{C}}$ & 0.02 & $<0.0001$ & $<0.0001$ \\
\hline Gl & $59 \cdot 6^{a}$ & 0.06 & $58 \cdot 2^{\mathrm{b}}$ & 0.06 & $55 \cdot 8^{\mathrm{C}}$ & 0.06 & $<0.0001$ & $<0.0001$ \\
\hline $\mathrm{GL}$ & $178 \cdot 3^{\mathrm{a}}$ & 0.5 & $171.4^{\mathrm{b}}$ & 0.5 & $161 \cdot 3^{\mathrm{c}}$ & 0.5 & $<0.0001$ & $<0.0001$ \\
\hline $\mathrm{Mg}(\mathrm{mg} / \mathrm{d})$ & $76 \cdot 8^{a}$ & 0.41 & $79 \cdot 4^{\mathrm{b}}$ & 0.4 & $84 \cdot 7^{\mathrm{C}}$ & 0.41 & $<0.0001$ & $<0.0001$ \\
\hline Fibre from cereals and grains $(\mathrm{g} / \mathrm{d})$ & $4 \cdot 2^{\mathrm{a}}$ & 0.03 & $4 \cdot 3^{\mathrm{b}}$ & 0.03 & $4 \cdot 0^{\mathrm{c}}$ & 0.03 & $<0.0001$ & 0.0062 \\
\hline Supplement users (\%) & & & & & & & & \\
\hline Multinutrients & & & & & & & 0.1482 & 0.0549 \\
\hline$n$ & & & & & & & & \\
\hline$\%$ & & & & & & & & \\
\hline Multinutrients or vitamin supplements§ & & & & & & & 0.0177 & 0.0048 \\
\hline$n$ & & & & & & & & \\
\hline$\%$ & & & & & & & & \\
\hline Food groupll & & & & & & & & \\
\hline Total vegetable $(\mathrm{g} / \mathrm{d})$ & $41 \cdot 8^{a}$ & 1.24 & $59 \cdot 1^{b}$ & 1.23 & $95 \cdot 9 c^{c}$ & $1 \cdot 24$ & $<0.0001$ & $<0.0001$ \\
\hline White rice $(\mathrm{g} / \mathrm{d})$ & $661 \cdot 2^{\mathrm{a}}$ & 3.63 & $609 \cdot 4^{\mathrm{b}}$ & 3.59 & $548 \cdot 4 c^{c}$ & 3.63 & $<0.0001$ & $<0.0001$ \\
\hline Dairy products $(\mathrm{g} / \mathrm{d})$ & $72 \cdot 7^{\mathrm{a}}$ & 2.75 & $86 \cdot 9^{\mathrm{b}}$ & $2 \cdot 73$ & $100 \cdot 9 c^{c}$ & $2 \cdot 76$ & $<0.0001$ & $<0.0001$ \\
\hline Red meat $(\mathrm{g} / \mathrm{d})$ & $13 \cdot 7$ & 0.51 & $13 \cdot 2$ & 0.50 & 13.5 & 0.51 & 0.7926 & 0.7683 \\
\hline Processed meat $(\mathrm{g} / \mathrm{d})$ & 0.2 & 0.03 & 0.2 & 0.03 & 0.2 & 0.03 & 0.8650 & 0.7462 \\
\hline Sweetened beverages $(\mathrm{g} / \mathrm{d})$ & $12 \cdot 6^{\mathrm{a}}$ & 1.01 & $8 \cdot 6^{\mathrm{b}}$ & 1.00 & $6 \cdot 1^{b}$ & 1.01 & $<0.0001$ & $<0.0001$ \\
\hline Coffee $(\mathrm{g} / \mathrm{d})$ & $3.1^{\mathrm{a}}$ & 0.08 & $3.0^{\mathrm{b}}$ & 0.08 & $2 \cdot 7^{\mathrm{c}}$ & 0.08 & $<0.0001$ & $<0.0001$ \\
\hline Dietary quality score & & & & & & & & \\
\hline KAHEIף & $31.5^{a}$ & 0.16 & $34.0^{\mathrm{b}}$ & 0.16 & $36 \cdot 4^{\mathrm{c}}$ & $0 \cdot 16$ & $<0.0001$ & $<0.0001$ \\
\hline
\end{tabular}

KAHEI, Korean Alternative Healthy Eating Index.

a,b,c Values within a row with unlike superscript letters were significantly different among the groups by Tukey's multiple-comparison test.

${ }^{*}$ High school graduation or longer ( $\geq 12$ years of education).

$\dagger \geq 3$ times/week and $30 \mathrm{~min} / \mathrm{session}$.

$\ddagger$ All nutrients were total energy-adjusted values.

$\S$ Vitamin supplements included supplements of vitamin $A$, vitamin $C, \beta$-carotene, vitamin $B$ complexes and vitamin $E$.

II Age- and total energy-adjusted means for food groups using general linear model.

II KAHEI was the modified alternative healthy eating index based on Korean Dietary Reference Intakes. 
Table 2. Type 2 diabetes (T2D) according to tertiles (T) of energy-adjusted folate intake (Incidence rate ratios (IRR) and $95 \%$ confidence intervals)

\begin{tabular}{|c|c|c|c|c|c|c|c|c|c|c|c|c|}
\hline \multirow[b]{4}{*}{ Variables } & \multicolumn{12}{|c|}{ Dietary folate intake $(\mu \mathrm{g} / \mathrm{d})$} \\
\hline & \multicolumn{5}{|c|}{ Men $(n$ 2693) } & \multicolumn{6}{|c|}{ Women $(n$ 4640) } & \multirow[b]{3}{*}{$P_{\text {trend }}{ }^{*}$} \\
\hline & \multirow{2}{*}{$\frac{\mathrm{T} 1}{\text { Reference }}$} & \multicolumn{2}{|r|}{ T2 } & \multicolumn{2}{|r|}{ Т3 } & \multirow[b]{2}{*}{$P_{\text {trend }}{ }^{*}$} & \multirow{2}{*}{$\frac{\mathrm{T} 1}{\text { Reference }}$} & \multicolumn{2}{|r|}{ T2 } & \multicolumn{2}{|r|}{ Т3 } & \\
\hline & & IRR & $95 \% \mathrm{Cl}$ & IRR & $95 \% \mathrm{Cl}$ & & & IRR & $95 \% \mathrm{Cl}$ & IRR & $95 \% \mathrm{Cl}$ & \\
\hline \multicolumn{13}{|l|}{ Baseline consumption $†$} \\
\hline Cases/person years & $36 / 3529$ & \multicolumn{2}{|c|}{$45 / 3665$} & \multicolumn{2}{|c|}{$47 / 3695$} & & $74 / 6177$ & \multicolumn{2}{|c|}{$64 / 6238$} & \multicolumn{2}{|c|}{$53 / 6441$} & \\
\hline Age-adjusted model & 1.00 & 1.20 & $0.78,1.86$ & 1.24 & $0.81,1.92$ & 0.3346 & 1.00 & 0.88 & $0.64,1.23$ & 0.73 & $0.51,1.03$ & 0.0726 \\
\hline Multivariable model 1 & 1.00 & 1.22 & $0.79,1.86$ & $1 \cdot 10$ & $0.72,1.67$ & 0.7301 & 1.00 & 0.74 & $0.53,1.04$ & 0.59 & $0.41,0.83$ & 0.0031 \\
\hline Multivariable model 2 & 1.00 & 1.22 & $0.79,1.88$ & 1.10 & $0.71,1.69$ & 0.7562 & 1.00 & 0.77 & $0.55,1.07$ & 0.63 & $0.43,0.93$ & 0.0192 \\
\hline Multivariable model 3 & 1.00 & $1 \cdot 28$ & $0.82,1.99$ & $1 \cdot 18$ & $0 \cdot 70,2 \cdot 01$ & 0.5815 & 1.00 & 0.73 & $0.52,1.02$ & 0.57 & $0.38,0.87$ & 0.0085 \\
\hline \multicolumn{13}{|l|}{ Average consumption $\ddagger$} \\
\hline Cases/person years & $39 / 3424$ & \multicolumn{2}{|c|}{$45 / 3848$} & \multicolumn{2}{|c|}{$44 / 3618$} & & $82 / 6027$ & \multicolumn{2}{|c|}{$53 / 6502$} & \multicolumn{2}{|c|}{$56 / 6327$} & \\
\hline Age-adjusted model & 1.00 & 1.03 & $0.67,1.58$ & 1.07 & $0.70,1.64$ & 0.7587 & 1.00 & 0.62 & $0.44,0.87$ & 0.69 & $0.49,0.97$ & 0.0439 \\
\hline Multivariable model 1 & 1.00 & 1.05 & $0.69,1.59$ & 0.96 & $0.63,1.45$ & 0.8059 & 1.00 & 0.58 & $0.41,0.82$ & 0.58 & $0.41,0.81$ & 0.0030 \\
\hline Multivariable model 2 & 1.00 & 1.04 & $0.68,1.58$ & 0.94 & $0.61,1.45$ & 0.7461 & 1.00 & 0.61 & $0.43,0.86$ & 0.68 & $0.47,0.98$ & 0.0421 \\
\hline Multivariable model 3 & 1.00 & 1.09 & $0.70,1.68$ & 1.04 & $0.62,1.76$ & 0.8958 & 1.00 & 0.55 & $0.39,0.79$ & 0.64 & $0.43,0.95$ & 0.0244 \\
\hline
\end{tabular}

AHEI, Alternative Healthy Eating Index.

* $P$ values for linear trend were obtained by imputing the median value of each tertile and treating it as a continuous variable using a modified Poisson regression with a robust error estimator.

† The multivariable model 1 for baseline consumption, the multivariable model 1 included age (years), higher education level (yes/no), regular exercise (yes/no), waist circumference $(\mathrm{cm})$, total energy intake $(\mathrm{kJ} / \mathrm{d})$ and baseline fasting blood glucose $(\mathrm{mg} / \mathrm{dl})$ in both men and women and additionally adjusted for smoking status (never/former/current) in men and current smoking (yes/no), alcohol consumption ( $\mathrm{g} / \mathrm{d}$ ) in women. In addition to model 1, the model 2 included animal Fe (mg/d), glycaemic load and Mg intake (mg/d) in both men and women and additionally adjusted for fibre from cereals and grains $(\mathrm{g} / \mathrm{d})$ for women. The mode 13 was additionally adjusted for total vegetables $(\mathrm{g} / \mathrm{d})$, dairy products $(\mathrm{g} / \mathrm{d})$, sweetened beverages $(\mathrm{g} / \mathrm{d})$, coffee consumption $(\mathrm{g} / \mathrm{d})$ and AHEI for men and women, as well as variables in the model 2 and additionally adjusted for red meat $(\mathrm{g} / \mathrm{d})$ in men. ‡ For average consumption, the multivariable model 1 included age (years), higher education level (yes/no), regular exercise (yes/no), waist circumference (cm) and baseline fasting blood glucose ( $\mathrm{mg} / \mathrm{dl}$ ) in both men and women and additionally included smoking status (never/former/current) in men and total energy intake (kJ/d), current smoking (yes/no) and alcohol consumption ( $\mathrm{g} / \mathrm{d}$ ) in women. In addition to model 1, the model 2 included Fe form animal food (mg/d), glycaemic load and Mg intake (mg/d) in both men and women and additionally adjusted for fibre from cereals and grains $(\mathrm{g} / \mathrm{d})$ for women. The mode1 3 was additionally adjusted for total vegetables $(\mathrm{g} / \mathrm{d})$, dairy products $(\mathrm{g} / \mathrm{d})$, sweetened beverages $(\mathrm{g} / \mathrm{d})$, coffee consumption $(\mathrm{g} / \mathrm{d})$ and AHEI for men and women, as well as variables in the model 2 and additionally adjusted for red meat $(\mathrm{g} / \mathrm{d})$ in men.

characteristics of participants as potential confounders by sex. The median value of baseline folate intakes among men and women were $369 \cdot 7$ and $333.7 \mu \mathrm{g} / \mathrm{d}$, respectively. Compared with T1, the higher proportion of participants in T3 had higher education levels, performed regular exercise, IFG and consumed multinutrients and/or vitamins in both men and women, whereas a lower proportion in T3 was current smoker. Participants in T3 had also higher mean WC and FBG in both men and women. Among dietary factors, the higher average consumptions in T3 compared with T1 were found in protein, fat, Fe from animal sources, $\mathrm{Mg}$, total vegetables, dairy products and KAHEI in both men and women, whereas the lower consumptions in T3 were found in energy, carbohydrate, glycaemic index, glycaemic load, white rice, sweet beverages and coffee consumption. We found that women in T3 were younger and had higher alcohol consumption and lower fibre from cereals and grains and men in T3 had lower processed meat. There were similar trends in these characteristics according to tertile of average dietary folate intake (online Supplementary Table S1).

Table 2 displays the IRR and 95\% CI according to dietary folate intake. For 29745 (sD 1.96) person years of follow-up, 319 participants developed de novo diabetes. Baseline folate intake was inversely associated with T2D risk in T3 compared with T1 after adjustment for all multiple confounding factors (IRR $=0.57$; $95 \%$ CI $0.38,0.87, P_{\text {for trend }}=0.0085$ ) among women. This inverse association was similar when the average folate consumption was used $\left(\mathrm{IRR}=0.64 ; 95 \% \mathrm{CI} 0.43,0.95, P_{\text {for trend }}=\right.$ $0 \cdot 0244)$. However, there was no association between folate and diabetes among men.
Table 3 shows the associations of dietary folate intake with T2D incidence in NFG and IFG group. Higher dietary intake of folate was associated with lower T2D risk both in NFG (IRR= $0 \cdot 32 ; 95 \%$ CI $0.13,0.81, P_{\text {for trend }}=0.0118$ for baseline dietary folate intake) and IFG group (IRR $=0.61 ; 95 \%$ CI $0.37,0.98$, $P_{\text {for trend }}=0.0382$ for average dietary folate intake) among women $\left(P_{\text {for }}\right.$ interaction $=0.0917$ for baseline folate intake; $P_{\text {for interaction }}=0.7653$ for average consumption), but not among men $\left(P_{\text {for interaction }}>0 \cdot 05\right)$.

Consumption of multinutrients and/or vitamins supplements was more prevalent in the higher tertile groups of both baseline and average folate consumption (Table 1 and online Supplementary Table S1), and supplement users might have special behaviours affecting their risk of developing de novo diabetes. Therefore, we further analysed the association between dietary folate intake and risk of developing diabetes after excluding users of multinutrients or multinutrients and/or vitamins (Table 4). Both baseline and average dietary folate intake were inversely associated with T2D in multinutrient non-users $\quad(\mathrm{IRR}=0.60 ; 95 \%$ CI 0.39 , 0.93; $P_{\text {for }}$ trend $=0.0213$ in T3 for baseline consumption; IRR = $0.65 ; 95 \%$ CI $0.43,0.996 ; \quad P_{\text {for }}$ trend $=0.0401$ in T3 for average consumption) among women. There was also an inverse association between dietary folate intake and T2D incidence in multinutrient and/or vitamin non-users ( $P_{\text {for trend }}=0.0453$ for baseline consumption and 0.0786 for average consumption) among women, although the result was not statistically significant in the analysis using average consumption. 
Table 3. Type 2 diabetes (T2D) according to tertiles (T) of dietary folate in normal fasting glucose (NFG) and impaired fasting glucose (IFG) group (Adjusted incidence rate ratios (IRR) and 95\% confidence intervals)

\begin{tabular}{|c|c|c|c|c|c|c|c|c|c|c|c|c|}
\hline \multirow[b]{4}{*}{ Variables } & \multicolumn{12}{|c|}{ Dietary folate intake $(\mu \mathrm{g} / \mathrm{d})$} \\
\hline & \multicolumn{5}{|c|}{ Men ( $n$ 2693) } & & \multicolumn{5}{|c|}{ Women $(n$ 4640) } & \multirow[b]{3}{*}{$P_{\text {trend }}{ }^{*}$} \\
\hline & \multirow{2}{*}{$\frac{\mathrm{T} 1}{\text { Reference }}$} & \multicolumn{2}{|r|}{ T2 } & \multicolumn{2}{|r|}{ Т3 } & \multirow[b]{2}{*}{$P_{\text {trend }}{ }^{*}$} & \multirow{2}{*}{$\frac{\mathrm{T} 1}{\text { Reference }}$} & \multicolumn{2}{|r|}{ T2 } & \multicolumn{2}{|r|}{ Т3 } & \\
\hline & & IRR & $95 \% \mathrm{Cl}$ & IRR & $95 \% \mathrm{Cl}$ & & & IRR & $95 \% \mathrm{Cl}$ & IRR & $95 \% \mathrm{Cl}$ & \\
\hline \multicolumn{13}{|l|}{ Baseline consumption $†$} \\
\hline NFG $(n 5125)$ & 593 & \multirow{2}{*}{\multicolumn{2}{|c|}{$\begin{array}{c}571 \\
13 / 2393\end{array}$}} & \multicolumn{2}{|r|}{536} & & 1167 & \multicolumn{2}{|c|}{1131} & \multicolumn{2}{|r|}{1127} & \\
\hline Cases/person years & 13/2397 & & & \multicolumn{2}{|c|}{$11 / 2228$} & & $24 / 4748$ & \multicolumn{2}{|c|}{$20 / 4647$} & \multicolumn{2}{|c|}{$9 / 4782$} & \\
\hline Multivariable model & 1.00 & 1.00 & $0.43,2.33$ & 1.05 & $0.38,2.91$ & 0.9300 & 1.00 & 0.89 & $0.48,1.65$ & 0.32 & $0.13,0.81$ & 0.0118 \\
\hline IFG $(n$ 2280) & 304 & \multicolumn{2}{|c|}{$\begin{array}{c}327 \\
32 / 1272\end{array}$} & \multirow{2}{*}{\multicolumn{2}{|c|}{$\begin{array}{c}327 \\
36 / 1467\end{array}$}} & & 379 & \multicolumn{2}{|c|}{416} & \multirow{2}{*}{\multicolumn{2}{|c|}{$\begin{array}{c}420 \\
44 / 1659\end{array}$}} & \\
\hline Cases/person years & $23 / 1132$ & \multicolumn{2}{|c|}{$32 / 1272$} & & & & $50 / 1429$ & \multicolumn{2}{|c|}{$44 / 1591$} & & & \\
\hline Multivariable model & 1.00 & 1.44 & $0.83,2.51$ & 1.35 & $0.71,2.56$ & 0.4153 & 1.00 & 0.68 & $0.45,1.02$ & 0.64 & $0.39,1.04$ & 0.0772 \\
\hline \multicolumn{13}{|l|}{ Average consumption $\ddagger$} \\
\hline NFG $(n$ 5125) & 592 & & 564 & & 544 & & 1163 & \multirow{2}{*}{\multicolumn{2}{|c|}{$\begin{array}{c}1136 \\
13 / 4813\end{array}$}} & \multirow{2}{*}{\multicolumn{2}{|c|}{$\begin{array}{c}1126 \\
14 / 4701\end{array}$}} & \\
\hline Cases/person years & 10/2322 & \multicolumn{2}{|c|}{$18 / 2489$} & \multicolumn{2}{|c|}{$9 / 2207$} & & $26 / 4663$ & & & & & \\
\hline Multivariable model & 1.00 & 1.87 & $0.78,4.49$ & 1.26 & $0.42,3.76$ & 0.6477 & 1.00 & 0.47 & $0.23,0.96$ & 0.64 & $0.31,1.33$ & 0.2303 \\
\hline IFG $(n$ 2280) & 305 & \multicolumn{2}{|r|}{334} & & 354 & & 383 & & 411 & & 421 & \\
\hline Cases/person years & 29/1101 & & $7 / 1358$ & & $5 / 1411$ & & $56 / 1364$ & & $0 / 1689$ & & 2/1626 & \\
\hline Multivariable model & 1.00 & 0.84 & $0.49,1.43$ & 1.06 & $0.59,1.90$ & 0.8304 & 1.00 & 0.56 & $0.38,0.83$ & 0.61 & $0.37,0.98$ & 0.0382 \\
\hline
\end{tabular}

$\mathrm{KAHEI}$, Korean Alternative Healthy Eating Index.

* $P$ values for linear trend were obtained by imputing the median value of each tertile and treating it as a continuous variable using a modified Poisson regression with a robust error estimator.

† The multivariable model for baseline dietary folate consumption was adjusted for age (years), higher education level (yes/no), smoking status (never/former/current), regular exercise (yes/no), waist circumference $(\mathrm{cm})$, total energy intake $(\mathrm{kJ} / \mathrm{d})$, baseline fasting blood glucose $(\mathrm{mg} / \mathrm{dl})$, Fe form animal food (mg/d), glycaemic load, Mg intake ( $\mathrm{mg} / \mathrm{d})$, total vegetables $(\mathrm{g} / \mathrm{d})$, dairy products $(\mathrm{g} / \mathrm{d})$, red meat $(\mathrm{g} / \mathrm{d})$, sweetened beverages $(\mathrm{g} / \mathrm{d})$, coffee consumption $(\mathrm{g} / \mathrm{d})$ and KAHEl in men and for age $(\mathrm{years})$, higher education level $(\mathrm{yes} /$ no), current smoking (yes/no), alcohol consumption ( $\mathrm{g} / \mathrm{d})$, regular exercise (yes/no), waist circumference $(\mathrm{cm})$, total energy intake $(\mathrm{kJ} / \mathrm{d})$, baseline fasting blood glucose $(\mathrm{mg} / \mathrm{dl})$, Fe form animal food $(\mathrm{mg} / \mathrm{d})$, glycaemic load, Mg intake $(\mathrm{mg} / \mathrm{d})$, fibre from cereals and grains $(\mathrm{g} / \mathrm{d})$, total vegetables $(\mathrm{g} / \mathrm{d})$, dairy products $(\mathrm{g} / \mathrm{d})$, sweetened beverages $(\mathrm{g} / \mathrm{d})$, coffee consumption $(\mathrm{g} / \mathrm{d})$ and $\mathrm{KAHEI}$ in women.

‡ The multivariable model for average dietary folate consumption was adjusted for age (years), higher education level (yes/no), smoking status (never/former/current), regular exercise (yes/no), waist circumference $(\mathrm{cm})$, baseline fasting blood glucose $(\mathrm{mg} / \mathrm{dl})$, Fe form animal food $(\mathrm{mg} / \mathrm{d})$, glycaemic load, Mg intake $(\mathrm{mg} / \mathrm{d})$, total vegetables $(\mathrm{g} / \mathrm{d})$, dairy products $(\mathrm{g} / \mathrm{d})$, red meat $(\mathrm{g} / \mathrm{d})$, sweetened beverages $(\mathrm{g} / \mathrm{d})$, coffee consumption (g/d) and KAHEl in men and for age (years), higher education level (yes/no), current smoking (yes/ $\mathrm{no})$, alcohol consumption (g/d), regular exercise (yes/no), waist circumference $(\mathrm{cm})$, total energy intake $(\mathrm{kJ} / \mathrm{d})$, baseline fasting blood glucose (mg/dl), Fe form animal food $(\mathrm{mg} / \mathrm{d})$, glycaemic load, Mg intake (mg/d), fibre from cereals and grains ( $\mathrm{g} / \mathrm{d})$, total vegetables $(\mathrm{g} / \mathrm{d})$, dairy products $(\mathrm{g} / \mathrm{d})$, sweetened beverages $(\mathrm{g} / \mathrm{d})$, coffee consumption $(\mathrm{g} / \mathrm{d})$ and $\mathrm{KAHEl}$ in women.

\section{Discussion}

To our knowledge, this prospective cohort study is the first study to evaluate the association between folate intake and the risk of developing diabetes. During the follow-up period, there was the inverse association between dietary folate intake and diabetes incidence risk among women. These inverse associations were found in both NFG group and IFG group among women.

There was no prospective cohort study to be directly compare with our finding of an inverse association between dietary folate intake and T2D risk, although several studies using folic acid supplementation ${ }^{(31)}$ with high doses of folic acid comparing to dietary intake. A meta-analysis reported that B vitamin supplementation including folate did not reduce the risk of vascular diseases ${ }^{(9)}$. However, a short-term intervention study in healthy overweight participants ( $n$ 60) revealed that folic acid supplementation $(2.5 \mathrm{mg} / \mathrm{d}$ for 3 months $)$ reduced FBG level and improved insulin sensitivity ${ }^{(31)}$. The improvements of insulin resistance and endothelial dysfunction were accompanied by reduced Hcy level after 6 months of folic acid administration $(5 \mathrm{mg} / \mathrm{d})$ with vitamin $\mathrm{B}_{12}(500 \mu \mathrm{g} / \mathrm{d})$ in $\mathrm{T} 2 \mathrm{D}$ patients $(n 50)^{(32)}$. In a meta-analysis of 183 diabetic patients from four studies, folic acid supplementation was also associated with better glycaemic control than placebo treatment ${ }^{(33)}$. MTHFR is the key rate-limiting enzyme required for the conversion of dietary folate to 5-methyltetrahydrofolate which is the methyl group donor required to convert Hcy to methionine in vivo ${ }^{(34)}$. A meta-analysis of studies on T2D associated with Hcy and the MTHFR and mendelian randomisation of the MTHFR gene suggested that the potential gene-environment interactions involved in the development of T2D and that the associations of Hcy with T2D may be modified by environmental factors such as dietary folate intake ${ }^{(18)}$. The results of our prospective observational study among adult participants without T2D diagnosed by physician or FBG $(\geq 126 \mathrm{mg} / \mathrm{dl}$; $7.0 \mathrm{mmol} / \mathrm{l})(n$ 7333) were consistent with the results of the above intervention study, although some but not all trials have revealed a significant beneficial effect of folic acid supplementation on glucose metabolism ${ }^{(35)}$.

Two different dietary folate intakes, the baseline and the average folate consumption were used in the analyses. The average folate consumption was calculated with intake data from the baseline until just before the censoring time or end point to reduce measurement error and/or to better represent long-term dietary patterns ${ }^{(36)}$. In the present study, it was difficult to assert which intake of baseline and average folate was better associated with T2D incidence and whether averaging folate intakes reduced measurement error. Therefore, further prospective cohort studies using repeated measures of dietary folate are needed to confirm this inverse relationship of dietary folate intake. 
Table 4. Type 2 diabetes (T2D) according to tertiles (T) of energy-adjusted folate intake among supplement non-users (Adjusted incidence rate ratios (IRR) and $95 \%$ confidence intervals)

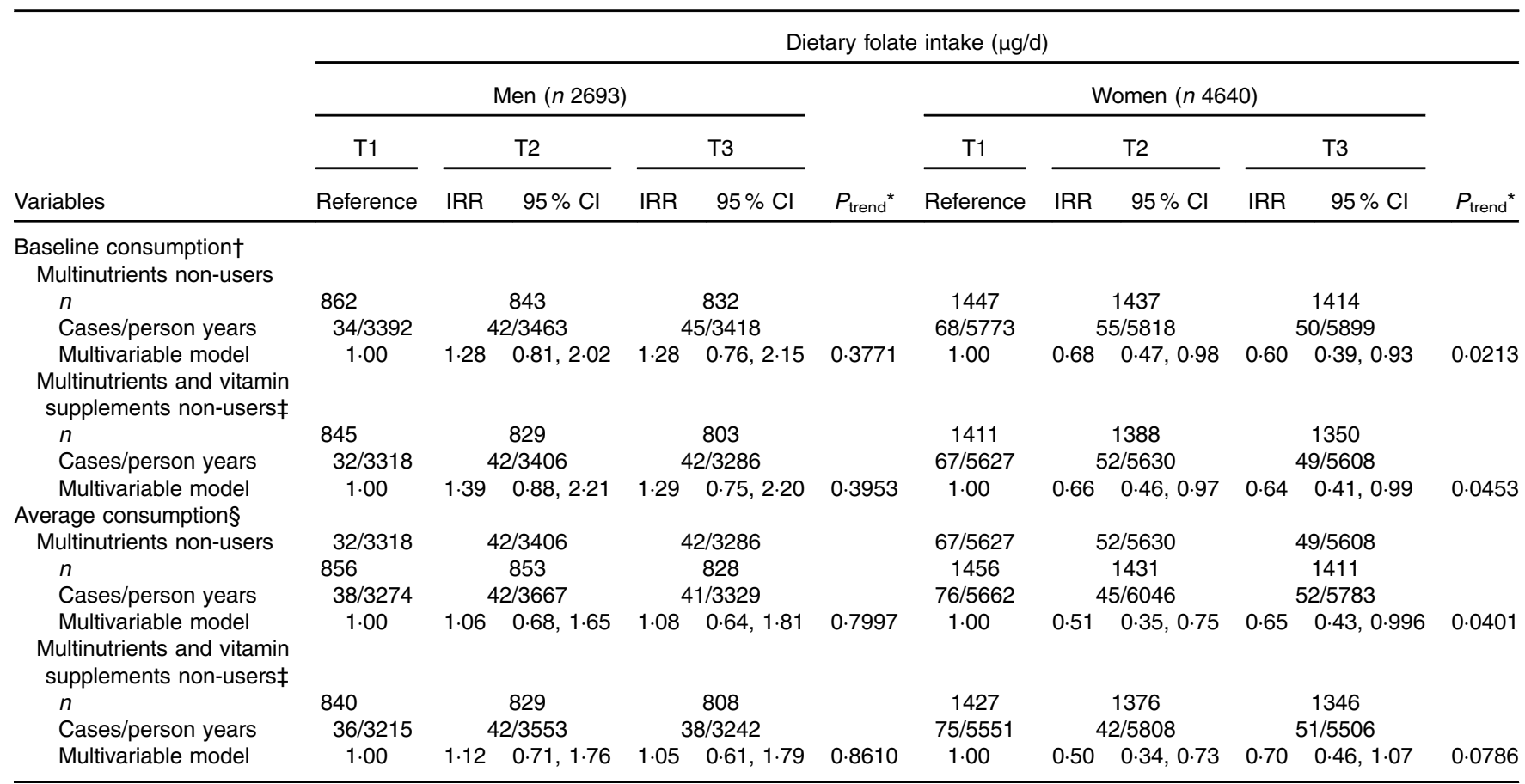

KAHEI, Korean Alternative Healthy Eating Index.

${ }^{*} P$ values for linear trend were obtained by imputing the median value of each tertile and treating it as a continuous variable using a modified Poisson regression with a robust error estimator.

† The multivariable model for baseline dietary folate consumption was adjusted for age (years), higher education level (yes/no), smoking status (never/former/current), regular exercise (yes/no), waist circumference $(\mathrm{cm})$, total energy intake $(\mathrm{kJ} / \mathrm{d})$, baseline fasting blood glucose $(\mathrm{mg} / \mathrm{dl})$, Fe form animal food $(\mathrm{mg} / \mathrm{d})$, glycaemic load, Mg intake $(\mathrm{mg} / \mathrm{d})$, total vegetables $(\mathrm{g} / \mathrm{d})$, dairy products $(\mathrm{g} / \mathrm{d})$, red meat $(\mathrm{g} / \mathrm{d})$, sweetened beverages $(\mathrm{g} / \mathrm{d})$, coffee consumption $(\mathrm{g} / \mathrm{d})$ and KAHEI in men and for age $(\mathrm{years})$, higher education level (yes/ no), current smoking (yes/no), alcohol consumption (g/d), regular exercise (yes/no), waist circumference (cm), total energy intake (kJ/d), baseline fasting blood glucose (mg/dl), Fe form animal food (mg/d), glycaemic load, Mg intake $(\mathrm{mg} / \mathrm{d})$, fibre from cereals and grains $(\mathrm{g} / \mathrm{d})$, total vegetables $(\mathrm{g} / \mathrm{d})$, dairy products $(\mathrm{g} / \mathrm{d})$, sweetened beverages $(\mathrm{g} / \mathrm{d})$, coffee consumption $(\mathrm{g} / \mathrm{d})$ and $\mathrm{KAHEl}$ in women.

‡ Vitamin supplements were supplements of vitamin $\mathrm{A}, \beta$-carotene, vitamin $\mathrm{C}$, vitamin $\mathrm{E}$ and vitamin $\mathrm{B}$ complexes

$\S$ The multivariable model for average dietary folate consumption was adjusted for age (years), higher education level (yes/no), smoking status (never/former/current), regular exercise (yes/no), waist circumference $(\mathrm{cm})$, baseline fasting blood glucose $(\mathrm{mg} / \mathrm{dll})$, Fe form animal food $(\mathrm{mg} / \mathrm{d})$, glycaemic load, Mg intake (mg/d), total vegetables $(\mathrm{g} / \mathrm{d})$, dairy products $(\mathrm{g} / \mathrm{d})$, red meat $(\mathrm{g} / \mathrm{d})$, sweetened beverages $(\mathrm{g} / \mathrm{d})$, coffee consumption $(\mathrm{g} / \mathrm{d})$ and $\mathrm{KAHEl}$ in men and for age (years), higher education level (yes/no), current smoking (yes/no), alcohol consumption (g/d), regular exercise (yes/no), waist circumference $(\mathrm{cm})$, total energy intake $(\mathrm{kJ} / \mathrm{d})$, baseline fasting blood glucose (mg/dl), Fe form animal food $(\mathrm{mg} / \mathrm{d})$, glycaemic load, $\mathrm{Mg}$ intake $(\mathrm{mg} / \mathrm{d})$, fibre from cereals and grains $(\mathrm{g} / \mathrm{d})$, total vegetables $(\mathrm{g} / \mathrm{d})$, dairy products $(\mathrm{g} / \mathrm{d})$, sweetened beverages $(\mathrm{g} / \mathrm{d})$, coffee consumption $(\mathrm{g} / \mathrm{d})$ and $\mathrm{KAHEI}$ in women.

T2D is a chronic non-communicable disease characterised by $\beta$-cell failure in the setting of insulin resistance. Although the mechanisms which folate reduces the risk of diabetes are not clearly understood, one possible mechanism is that folate can reduce the level of Hcy, which may inhibit the insulinstimulated tyrosine phosphorylation of the insulin receptor $\beta$-subunit and its substrates, reduce the activity of the p85 regulatory subunit of phosphatidylinositol 3-kinase, and reduce insulin-stimulated glycogen synthesis ${ }^{(37)}$. Furthermore, it was reported that Hcy inhibits the activation of adenylate cyclase and protein kinase $\mathrm{C}$ pathways through induction of insulin secretion in pancreatic $\beta$ cells ${ }^{(38)}$. In addition, folate has been shown to ameliorate arsenic- and nicotine-induced oxidative damage in rat pancreatic tissue ${ }^{(39,40)}$. The main circulating metabolite of folate, 5-methyltetrahydrofolate, has prominent antioxidant activity ${ }^{(41)}$ and increases nitric oxide production ${ }^{(42)}$. Increased production of reactive $\mathrm{O}_{2}$ species from mitochondrial overstimulation and reactive nitrogen species from excess nitric oxide generation in $\beta$ cells may inhibit the electron transport chain and thus reduce energy production, damage DNA and promote the formation of advanced glycation end products, ultimately leading to $\beta$ cell dysfunction ${ }^{(43)}$. These findings may suggest that folate itself may influence the risk of diabetes, independent of its regulation of Hcy concentration.

Although we could not define prediabetes, it is worth to note the inverse association between dietary folate intake and T2D incidence risk even in IFG group, as well as in NFG group. To our best knowledge, there was no prospective cohort study considering NFG and/or IFG status on the association between folate intake and T2D incidence.

We unexpectedly found the higher FBG level in T3 of folate intake compared with $\mathrm{T} 1$. It may be due to the higher proportion of IFG group, which had higher folate intake than NFG group (data not shown; all $P$ values $<0.05$ for both baseline folate and average folate in men and women), in T3 of dietary folate intake compared with T1. The higher folate intake in IFG was likely because more participants in IFG group have been alerted to high FBG and thus have been already changed their diets compared with NFG group. Furthermore, the increasing pattern of T2D risk in IFG group from T1 of the baseline folate intake to T3 appeared. The likelihood of T2D occurrence among participants with IFG compared with NFG was a predictable result. 
The increasing pattern from $\mathrm{T} 1$ to $\mathrm{T} 3$ may be also due to already changed diet among participants with high FBG.

We found the sex difference in the association between dietary folate intake and T2D incidence risk ( $P_{\text {for interaction }}=$ 0.0411 for baseline folate intake; $P_{\text {for interaction }}=0.0471$ for average folate intake), although foods highly providing dietary folate and contributing variation of total folate intake $\left(R^{2}\right)$ were almost same between men and women (online Supplementary Table S2). In the present study, we could not assert why there was sex difference in the association between dietary folate intake and T2D incidence risk. However, it may be partially explained by that the higher concentration of serum Hcy, the lower concentration of serum folate and vitamin $\mathrm{B}_{12}$ among men than women reported in our previous study among adults in a community of the present study areas ${ }^{(44)}$, although dietary folate intake was higher among men than women. Another previous study on B vitamins and Hcy level among Hispanic men and women suggested the possibility that the higher concentration in men even though controlling for dietary B vitamins intakes may be due to sex differences in Hcy metabolic regression and that the higher intake of $\mathrm{B}$ vitamins including folate may be needed to lower Hcy in men than in women ${ }^{(45)}$.

There were some limitations to the present study. This study was an observational prospective cohort study and the development of diabetes may be influenced by lifestyle changes ${ }^{(46)}$. We included many potential confounders correlated with T2D ${ }^{(12-17,25)}$. However, there may be residual confounding by unmeasured confounding factors such as Hcy level. In addition, confounding from multiple food sources was still possible. Because higher daily intake of folate tended to be related to some food that may have beneficial effects on T2D such as green leafy vegetable, whole grain and dairy products (online Supplementary Fig. S1). Therefore, the results from the present study should be interpreted in the context of the whole dietary patterns. Second, we could not confirm the association between folate and T2D with biomarker such as serum/erythrocyte folate. Third, follow-up loss may lead to biased results. Although participants who retained until the second revisit were likely to be relatively healthier comparing to participants who lost to follow-up after baseline and the first revisit, the findings from the analyses using only data until the first revisit were very similar to this study (data not shown). Furthermore, in the sensitivity analysis of the assignment methods of follow-up time to evaluate the effect of follow-up losses in the exposure group (online Supplementary Table S3); (1) the smallest denominator and (2) the largest denominator, the significant inverse associations appeared in both assignment methods of follow-up time. However, the problem resulting from the possibility that loss to follow-up was biased with respect to both exposure and outcome remains ${ }^{(23)}$. It is generally recognised that the validity of the statistical results derived from the study (such as risk ratios) may be affected when follow-up losses are uneven in both the exposure and outcome categories ${ }^{(47)}$. Thus, we also conducted household visit survey among subjects who did not participate in repeated examinations between 2015 and 2016 to check if there was a new T2D diagnosed by medical doctor and if taking medication. The follow-up period was different from the present study and T2D by FBG could not be included.
Considering non-participants in repeated examination (follow-up method in the present study) as loss to follow-up ${ }^{(47)}$, there was no significant follow-up bias according to tertiles of dietary folate intake (sex and age standardised follow-up bias, $P=0.2501$ in the entire participants; $P=0.3809$ in NFG group; $P=0.0646$ in IFG group). However, cohort studies following up over a longer period are traditionally subject to selection bias. This implies participants who are at risk are more likely to participate and continue being in the study. Even when the results are valid for the present cohort and present conditions, selection bias may influence the external validity and generalisability of the results. Fourth, self-report of physician-diagnosed diabetes and medication was not validated with medical record, although self-reported T2D was consistently responded between visits (92.4\%). In addition, the glycosylated $\mathrm{Hb}(\%)$ and the OGTT was not measured in the cohort and thus diabetes by OGTT could not be defined. Finally, there was limitation directly to explain the possible mechanism under the development of T2D without biomarkers such as folate and vitamin $\mathrm{B}_{12}$. Further long-term prospective studies with repeated measures of biomarkers are needed.

In conclusion, higher intake of folate may be associated with a lower risk of diabetes. In addition, this effect is independent of previously identified risk factors of diabetes and other nutrient factors. Further epidemiological and experimental studies are required to confirm the effect of folate on diabetes risk.

\section{Acknowledgements}

The authors thank the Korea Centers for Disease Control and Prevention for the administration of data.

This work was supported by the Research Program funded by the Korea Centers for Disease Control and Prevention (2004E71004-00, 2005-E71011-00, 2006-E71009-00, 2007-E71002-00, 2008-E71004-00, 2009-E71006-00, 2010-E71003-00, 2011E71002-00, 2012-E71007-00, 2013-E71008-00) and was supported by the National Research Foundation of Korea grant funded by the Korea government (Ministry of Science, ICT \& Future Planning) (no. 2016R1A2B2011352).

S. M. H. and H. W. W equally contributed to the design of this study and the data analysis and wrote the manuscript. S. Y. K. analysed data and was involved in interpreting the results. B.-Y. C., Y.-H. L., D. H. S., M.-H. S. B.-Y. C., and B. Y. C. were involved in data collection and management. M. K. K. designed the study and supervised all aspects of its implementation. All authors helped to conceptualise the study, interpret findings and review drafts of the manuscript.

The authors declare that there are no conflicts of interest.

\section{Supplementary material}

For supplementary material/s referred to in this article, please visit https://doi.org/10.1017/S0007114517003087

\section{References}

1. Ebara S (2017) Nutritional role of folate. Congenit Anom (Kyoto) 57, 138-141. 
2. Stover PJ (2009) Folate biochemical pathways and their regulation. In Folate in Health and Disease, 2nd edition, pp. 49-74 [LB Bailey, editor]. Gainesville, FL: CRC Press.

3. Bailey LB \& Caudil MA (2012) Folate. In Present Knowledge in Nutrition, tenth edition, pp. 321-342 [JW Erdman, IA Macdonald and SH Zeisel, editors]. Oxford: Wiley-Blackwell.

4. Wang Z-M, Zhou B, Nie Z-L, et al. (2012) Folate and risk of coronary heart disease: a meta-analysis of prospective studies. Nutr Metab Cardiovasc Dis 22, 890-899.

5. Chen J, Xu X, Liu A, et al. (2009) Folate and cancer. In Folate in Health and Disease, 2nd ed., pp. 205-233 [LB Bailey, editor]. Gainesville, FL: CRC Press.

6. Cooper C, Sommerlad A, Lyketsos CG, et al. (2015) Modifiable predictors of dementia in mild cognitive impairment: a systematic review and meta-analysis. Am J Psychiatry 172, 323-334.

7. Blom HJ \& Smulders Y (2011) Overview of homocysteine and folate metabolism. With special references to cardiovascular disease and neural tube defects. I Inherit Metab Dis 34, 75-81.

8. McCully KS (2009) Chemical pathology of homocysteine. IV. Excitotoxicity, oxidative stress, endothelial dysfunction, and inflammation. Ann Clin Lab Sci 39, 219-232.

9. Bailey LB, da Silva V, West AA, et al. (2013) Folate. In Handbook of Vitamins, p. 421, [J Zempleni, JW Suttie, JF Gregory III and PJ Stover, editors]. Gainesville, FL: CRC Press, Taylor \& Francis Group.

10. Grundy SM, Benjamin IJ, Burke GL, et al. (1999) Diabetes and cardiovascular disease. Circulation 100, 1134-1146.

11. Kato $N$ (2013) Insights into the genetic basis of type 2 diabetes. J Diabetes Investig 4, 233-244.

12. Agardh E, Allebeck P, Hallqvist J, et al. (2011) Type 2 diabetes incidence and socio-economic position: a systematic review and meta-analysis. Int J Epidemiol 40, 804-818.

13. Willi C, Bodenmann P, Ghali WA, et al. (2007) Active smoking and the risk of type 2 diabetes: a systematic review and metaanalysis. JAMA 298, 2654-2664.

14. Li X-H, Yu F-f, Zhou Y-H, et al. (2016) Association between alcohol consumption and the risk of incident type 2 diabetes: a systematic review and dose-response meta-analysis. Am J Clin Nutr 103, 818-829.

15. Jeon CY, Lokken RP, Hu FB, et al. (2007) Physical activity of moderate intensity and risk of type 2 diabetes. Diabetes Care 30, 744-752.

16. Vazquez G, Duval S, Jacobs DR Jr, et al. (2007) Comparison of body mass index, waist circumference, and waist/hip ratio in predicting incident diabetes: a meta-analysis. Epidemiol Rev 29, 115-128.

17. Ley SH, Hamdy O, Mohan V, et al. (2014) Prevention and management of type 2 diabetes: dietary components and nutritional strategies. Lancet 383, 1999-2007.

18. Huang $\mathrm{T}$, Ren J, Huang J, et al. (2013) Association of homocysteine with type 2 diabetes: a meta-analysis implementing Mendelian randomization approach. BMC Genomics 14, 867.

19. Ahn Y, Kwon E, Shim JE, et al. (2007) Validation and reproducibility of food frequency questionnaire for Korean genome epidemiologic study. Eur J Clin Nutr 61, 1435-1441.

20. Society TKN (2009) Food Values. Seoul: The Korean Nutrition Society.

21. Willett WC (2013) Nutritional Epidemiology, 3rd ed. New York: Oxford University Press.

22. American Diabetes Association (2014) Standards of medical care in diabetes - 2014. Diabetes care 2014; 37 (Suppl. 1): S14-S80 Diagnosis and classification of diabetes mellitus. Diabetes care 2014; 37 (Suppl. 1): S81-S90. Diabetes Care 37, $887-887$.
23. MacMahon B \& Pugh TF (1970) Epidemiology; Principles and Methods, 1st ed. Boston, MA: Little.

24. Anderson JW, Kendall CW \& Jenkins DJ (2003) Importance of weight management in type 2 diabetes: review with metaanalysis of clinical studies. J Am coll Nutr 22, 331-339.

25. McCullough ML, Feskanich D, Stampfer MJ, et al. (2002) Diet quality and major chronic disease risk in men and women: moving toward improved dietary guidance. Am J Clin Nutr 76, 1261-1271.

26. Zou G (2004) A modified Poisson regression approach to prospective studies with binary data. Am J Epidemiol 159, 702-706.

27. Callas PW, Pastides H \& Hosmer DW (1998) Empirical comparisons of proportional hazards, Poisson, and logistic regression modeling of occupational cohort data. Am J Ind Med 33, 33-47.

28. Lyle BJ, Mares-Perlman JA, Klein BE, et al. (1998) Supplement users differ from nonusers in demographic, lifestyle, dietary and health characteristics. J Nutr 128, 2355-2362.

29. Würtz AM, Hansen MD, Tjønneland A, et al. (2016) Substitutions of red meat, poultry and fish and risk of myocardial infarction. Br J Nutr 115, 1571-1578.

30. Ryu C \& Hong S (2015) $R$ Visualization, 1st ed. Seoul, South Korea: Insight.

31. Solini A, Santini E \& Ferrannini E (2006) Effect of short-term folic acid supplementation on insulin sensitivity and inflammatory markers in overweight subjects. Int J Obes (Lond) $\mathbf{3 0}$, 1197-1202.

32. Setola E, Monti LD, Galluccio E, et al. (2004) Insulin resistance and endothelial function are improved after folate and vitamin $\mathrm{B}_{12}$ therapy in patients with metabolic syndrome: relationship between homocysteine levels and hyperinsulinemia. Eur J Endocrinol 151, 483-489.

33. Sudchada P, Saokaew S, Sridetch S, et al. (2012) Effect of folic acid supplementation on plasma total homocysteine levels and glycemic control in patients with type 2 diabetes: a systematic review and meta-analysis. Diabetes Res Clin Pract 98, 151-158.

34. Jacques PF, Bostom AG, Williams RR, et al. (1996) Relation between folate status, a common mutation in methylenetetrahydrofolate reductase, and plasma homocysteine concentrations. Circulation 93, 7-9.

35. Song Y, Cook NR, Albert CM, et al. (2009) Effect of homocysteine-lowering treatment with folic Acid and B vitamins on risk of type 2 diabetes in women: a randomized, controlled trial. Diabetes 58, 1921-1928.

36. Hu FB, Stampfer MJ, Rimm E, et al. (1999) Dietary fat and coronary heart disease: a comparison of approaches for adjusting for total energy intake and modeling repeated dietary measurements. Am J Epidemiol 149, 531-540.

37. Najib S \& Sanchez-Margalet V (2001) Homocysteine thiolactone inhibits insulin signaling, and glutathione has a protective effect. J Mol Endocrinol 27, 85-91.

38. Patterson S, Flatt PR, Brennan L, et al. (2006) Detrimental actions of metabolic syndrome risk factor, homocysteine, on pancreatic beta-cell glucose metabolism and insulin secretion. J Endocrinol 189, 301-310.

39. Mukherjee S, Das D, Mukherjee M, et al. (2006) Synergistic effect of folic acid and vitamin $\mathrm{B}_{12}$ in ameliorating arsenicinduced oxidative damage in pancreatic tissue of rat. $J$ Nutr Biochem 17, 319-327.

40. Bhattacharjee A, Prasad SK, Pal S, et al. (2016) Synergistic protective effect of folic acid and vitamin $\mathrm{B}_{12}$ against nicotineinduced oxidative stress and apoptosis in pancreatic islets of the rat. Pharm Biol 54, 433-444.

41. Rezk BM, Haenen GR, van der Vijgh WJ, et al. (2003) Tetrahydrofolate and 5-methyltetrahydrofolate are folates with high 
antioxidant activity. Identification of the antioxidant pharmacophore. FEBS Lett 555, 601-605.

42. Stroes ES, van Faassen EE, Yo M, et al. (2000) Folic acid reverts dysfunction of endothelial nitric oxide synthase. Circ Res 86, 1129-1134.

43. Cerf ME (2013) Beta cell dysfunction and insulin resistance. Front Endocrinol (Lausanne) 4, 37.

44. Kim HJ, Kim MK, Kim JU, et al. (2010) Major determinants of serum homocysteine concentrations in a Korean population. J Korean Med Sci 25, 509-516.
45. Kim MK, Ordovas JM, Selhub J, et al. (2003) B vitamins and plasma homocysteine concentrations in an urban and rural area of Costa Rica. J Am Coll Nutr 22, 224-231.

46. Tuomilehto J, Lindström J, Eriksson JG, et al. (2001) Prevention of type 2 diabetes mellitus by changes in lifestyle among subjects with impaired glucose tolerance. $N$ Engl $J$ Med 344, 1343-1350.

47. Greenland S (1977) Response and follow-up bias in cohort studies. Am J Epidemiol 106, 184-187. 